\title{
Seleção de fungos micorrízicos arbusculares e ectomicorrízicos para simbioses eficientes com Acacia mangium willd
}

\section{Seletion of arbuscular mycorrhizal and ectomycorrhizal fungi for efficient symbiosis with Acacia mangium willd}

\author{
Guilherme Augusto Robles Angelini ${ }^{1 *}$; Orivaldo José Saggin Júnior²; \\ Eliane Maria Ribeiro da Silva ${ }^{2}$
}

\begin{abstract}
Resumo
Acacia mangium forma dois tipos de simbiose micorrízica, uma com fungos micorrízicos arbusculares (FMAs) e outra com fungos ectomicorrízicos (fECTOs). O presente trabalho teve o objetivo de selecionar diferentes espécies de FMAs e isolados de fECTOs para simbioses eficientes com A. mangium, que proporcionem mudas bem micorrizadas, noduladas e desenvolvidas. Experimentos foram instalados em casa de vegetação na Embrapa Agrobiologia, sendo um para a seleção de FMAs e outro para fECTOs, utilizando delineamento de blocos casualizados, com 5 repetições. Tratamentos foram espécies de FMAs (Acaulospora laevis, Acaulospora morrowiae, Entrophospora colombiana, Entrophospora contigua, Gigaspora margarita, Glomus clarum, Scutellospora calospora, Scutellospora heterogama, Scutellospora gilmorei e Scutellospora pellucida) ou isolados de fECTOs (UFSC Pt116; UFSC Pt24; UFSC Pt193; O 64-ITA6; UFSC Pt187 e O 40-ORS 7870). As espécies de FMAs que promoveram maior crescimento vegetativo, colonização micorrízica e apresentaram simbiose mais eficiente foram S. calospora, S. heterogama, S. gilmorei e A. morrowiae. Os fECTOs não demonstraram eficiência na promoção do crescimento, mas o isolado O64-ITA6 (Pisolithus tinctorius) proporcionou maior colonização micorrízica. Mudas de A. mangium são muito responsivas a inoculação de FMAs e dependem de elevada colonização micorrízica, entre 40 e $80 \%$, para obter benefícios relevantes da simbiose no crescimento e nodulação.

Palavras-chave: Eficiência simbiótica, colonização micorrízica, nodulação, benefício micorrízico, leguminosa arbórea
\end{abstract}

\begin{abstract}
Acacia mangium forms two kinds of mycorrhizal symbiosis, a arbuscular mycorrhizal fungi (AMFs) type and another with ectomycorrhizal fungi (fECTOs). The present study aimed to select different AMFs species and fECTOs isolates for effective symbiosis with A. mangium, which provide seedlings well colonized, nodulated and developed. Experiments were conducted in a greenhouse at Embrapa Agrobiology, one for AMF species selection and another for fECTOs, using a randomized block design with five replicates. Treatments were species AMFs (Acaulospora laevis, Acaulospora morrowiae, Entrophospora colombiana, Entrophospora contigua, Gigaspora margarita, Glomus clarum, Scutellospora calospora, Scutellospora heterogama, Scutellospora gilmorei and Scutellospora pellucida) or fECTOs isolated (UFSC Pt116; UFSC Pt24; UFSC Pt193; O 64-ITA6; UFSC Pt187 and O 40-ORS 7870). The AMFs species that promoted greater vegetative growth, mycorrhizal colonization and more effective symbioses were $S$. calospora, S. heterogama, S. gilmorei e A. morrowiae. The fECTOs
\end{abstract}

1 Dr. em Agronomia, Instituto de Agronomia, Dept ${ }^{\circ}$ de Fitotecnia, Universidade Federal Rural do Rio de Janeiro, UFRRJ/Embrapa Agrobiologia, Seropédica, RJ. E-mail: gara_agr@hotmail.com

2 Pesquisadores da Embrapa Agrobiologia, Seropédica, RJ. E-mail: saggin@cnpab.embrapa.br; eliane@cnpab.embrapa.br Autor para correspondência 
not demonstrated effectiveness in promoting growth, but the isolate O64-ITA6 (Pisolithus tinctorius) provided greater colonization. Seedlings of A. mangium have high responsiveness to inoculation with AMFs and depends on high root colonization, between 40 and $80 \%$, to obtain relevant benefits from symbiose over nodule formation and growth.

Key words: Symbiotic effectiveness, root colonization, nodulation, mycorrhizal benefit, leguminous tree

\section{Introdução}

A Acacia mangium tem sido utilizada para se obter uma rápida retomada da cobertura vegetal arbórea em áreas degradadas, propiciando o reinicio da sucessão florestal natural nessas áreas (FRANCO et al., 1995). Esta espécie destaca-se por formar três tipos de simbiose: a fixadora de nitrogênio atmosférico com rizóbio, a com fungos micorrízicos arbusculares (FMAs) e a com fungos ectomicorrízicos (fECTOs). Os FMAs e os fECTOs se diferenciam pelo filo, por suas características morfológicas, por suas estruturas de reprodução, pela morfologia da colonização radicular e por seus hospedeiros (SAGGIN JÚNIOR; SILVA, 2005). Apesar disto, algumas angiospermas arbóreas, como as do gênero Acacia, podem formar estes dois tipos de micorrizas.

Tanto os FMAs quanto os fECTOs trazem benefícios à comunidade vegetal e ao ambiente. Proporcionam aumento da superfície de absorção e do volume do solo explorado, promovendo melhoria na nutrição e hidratação das plantas, maior resistências a estresses biológicos e físico-químicos e melhora direta do solo por promover agregação e estabilidade dos agregados (SMITH; READ, 2008).

Desta forma, a inoculação dos fungos micorrízicos em mudas arbóreas destinadas para revegetação de áreas degradadas é uma tecnologia desenvolvida pela Embrapa (FRANCO et al., 1995), hoje bastante aplicada e importante por auxiliar e acelerar os processos de recuperação dos solos, ajudando no estabelecimento das mudas no campo e no restabelecimento da ciclagem de nutrientes. No caso dos FMAs, as relações fungoplanta são consideradas não específicas. No entanto, há forte evidência de variabilidade funcional entre as espécies de FMAS que resultam em respostas diferenciadas em absorção de $\mathrm{P}$, proteção contra patógenos e aumento da produtividade. Como consequência as respostas de uma planta a diferentes FMAs são muito diferenciadas e altamente específicas em determinada condição edafoambiental (KLIRONOMOS, 2000).

Já no caso do fECTOs, o grau de especificidade com o hospedeiro é bastante variável, particularmente entre as espécies fúngicas (BRUNS, BIDARTONDO; TAYLOR, 2002). Nos casos mais específicos, novas simbioses são iniciadas por propágulos dormentes que somente germinam quando estimulados por sinais das plantas hospedeiras específica. Porém, grande maioria das plantas ectomicorrízicas se associa a um grande número de fungos (BRUNS; BIDARTONDO; TAYLOR, 2002), havendo diferentes graus de especificidade.

Dentro deste contexto, para melhorar a produção de mudas de Acacia mangium e o seu desempenho em áreas degradadas torna-se importante avaliar as respostas de suas mudas quando inoculadas com diferentes FMAs e diferentes fECTOs. Desta maneira é possível selecionar combinações mais eficientes de Acacia mangium x isolado fúngico, obtendose maior crescimento da muda, planta mais bem nutrida e maior resistência e desenvolvimento póstransplante. Este tipo de resultado pode evoluir para a elaboração de um inoculante de múltiplos fungos selecionados, o que seria um passo importante no sentido de conseguir um biofertilizante micorrízico para uso comercial no Brasil.

Assim, o objetivo deste trabalho foi selecionar diferentes espécies de FMAs e isolados de fECTOs para simbioses eficientes com Acacia mangium, que proporcionem mudas bem micorrizadas, noduladas e desenvolvidas. 


\section{Material e Métodos}

O estudo foi desenvolvido em casa de vegetação no Centro Nacional de Pesquisa de Agrobiologia - Embrapa Agrobiologia, em Seropédica, RJ $\left(22^{\circ} 45^{\prime} 32,27^{\prime \prime}\right.$ Latitude Sul e $43^{\circ} 40^{\prime} 51,52^{\prime \prime}$ Longitude Oeste). A casa de vegetação possui luminosidade natural e temperatura ambiente resfriada por ventilação úmida quando ultrapassa $32^{\circ} \mathrm{C}$. O clima da região, pela classificação de Köppen, é Aw caracterizado como Tropical chuvoso com inverno seco.
Foram conduzidos dois experimentos de seleção de fungos micorrízicos para Acacia mangium entre fevereiro e julho de 2007, sendo um experimento com fungos micorrízicos arbusculares (FMAs) e outro com fungos ectomicorrízicos (fECTOs). Os tratamentos constaram de diferentes espécies ou isolados de fungos descritos na Tabela 1, acrescido de uma testemunha não inoculada. Em ambos experimentos o delineamento experimental foi blocos casualizados com 5 repetições.

Tabela 1. Espécies e isolados de fungos micorrízicos utilizados como tratamentos nos experimentos de seleção de simbiose eficiente para Acacia mangium.

\begin{tabular}{lcc}
\hline Espécie de fungo & Identificação do isolado & Coleção fornecedora \\
\hline & Experimento 1 (Seleção de FMAs $\left.{ }^{1}\right)$ & \\
Acaulospora laevis & A39 (IES-35) & COFMEA ${ }^{3}$ \\
Acaulospora morrowiae & A19 (CNPAB 019) & COFMEA \\
Entrophospora colombiana & A15 (CNPAB 015) & COFMEA \\
Entrophospora contigua & A28 (IES - 4 (b)) & COFMEA \\
Gigaspora margarita & A1 (CNPAB 001) & COFMEA \\
Glomus clarum & A5 (CNPAB 005) & COFMEA \\
Scutellospora calospora & A80 (CNPAB 038) & COFMEA \\
Scutellospora heterogama & A2 (CNPAB 002) & COFMEA \\
Scutellospora gilmorei & A13 (CNPAB 013) & COFMEA \\
Scutellospora pellucida & A70 (CNPAB 029) & COFMEA \\
\hline & Experimento 2 (Seleção de fECTOs $\left.{ }^{2}\right)$ & \\
Pisolithus microcarpus & UFSC Pt 116 & MIP/CCB/UFSC \\
Pisolithus microcarpus & UFSC Pt 187 & MIP/CCB/UFSC \\
Pisolithus tinctorius & UFSC Pt 24 & MIP/CCB/UFSC \\
Pisolithus tinctorius & O 64-ITA 6 & MIP/CCB/UFSC \\
Pisolithus sp & UFSC Pt 193 & MIP/CCB/UFSC \\
Pisolithus sp & O 40-ORS 7870 & MIP/CCB/UFSC \\
\hline
\end{tabular}

${ }^{1}$ FMAs: Fungos Micorrízicos Arbusculares

${ }^{2}$ fECTOS: Fungos Ectomicorrízicos

${ }^{3}$ COFMEA: Coleção de Fungos Micorrízicos Arbusculares da Embrapa Agrobiologia

${ }^{4}$ MIP/CCB/UFSC: Departamento de Microbiologia, Imunologia e Parasitologia (MIP) do Centro de Ciências Biológicas (CCB) da Universidade Federal de Santa Catarina (UFSC)

Fonte: Elaboração dos autores.

Os experimentos foram conduzidos em recipientes de $980 \mathrm{~mL}$, na forma de um tubete plástico com $32 \mathrm{~cm}$ de altura e $10 \mathrm{~cm}$ de diâmetro superior. Estes foram preenchidos com material de um Nitossolo Háplico Distrófico (Udult, segundo FAO, 2006), coletado a 22³2'11,9'” de Latitude Sul e $43^{\circ} 59^{\prime} 57,7^{\prime \prime}$ de Longitude Oeste. O material de solo foi seco ao ar, peneirado em malha de $2 \mathrm{~mm}$ e recebeu calagem na dose de $1,25 \mathrm{~g} \mathrm{~kg}^{-1}$ de calcário dolomítico de Poder Relativo de Neutralização Total 75\% (PRNT). O solo foi então autoclavado a $120{ }^{\circ} \mathrm{C}$, pressão de $1 \mathrm{~atm}$, por 60 minutos, em dois dias consecutivos. Deixou-se o solo estabilizar os teores de manganês por 15 dias após a autoclavagem 
para então preencher os recipientes. Nesta ocasião o solo apresentava $\mathrm{pH} \mathrm{H}_{2} \mathrm{O}(1: 2,5) 5,2 ; 2 \mathrm{mg} \mathrm{dm}^{-3} \mathrm{de}$ P; 49,8 $\mathrm{mg} \mathrm{dm}^{-3}$ de $\mathrm{K} ; 1,3 \mathrm{cmol}_{\mathrm{c}} \mathrm{dm}^{-3} \mathrm{de} \mathrm{Ca}^{2+} ; 0,4$ $\mathrm{cmol}_{\mathrm{c}} \mathrm{dm}^{-3}$ de $\mathrm{Mg}^{2+} ; 1,7 \mathrm{cmol}_{\mathrm{c}} \mathrm{dm}^{-3}$ de $\mathrm{Ca}+\mathrm{Mg} ; 0,6$ $\mathrm{cmol}_{\mathrm{c}} \mathrm{dm}^{-3}$ de $\mathrm{Al}$ e saturação por bases (V\%) 45.

A inoculação dos tratamentos fúngico foi realizada no orifício de plantio durante o transplante de sementes pré-germinadas nos dois experimentos (com radículas entre 2-10 $\mathrm{mm}$ ). Os inoculantes de FMAs foram multiplicados utilizando Brachiaria decumbens como planta hospedeira e na inoculação do experimento aplicou-se $3 \mathrm{~mL}$ de inoculante por orifício, quantidade esta suficiente para inocular em cada tratamento fúngico pelo menos 50 esporos de FMAs, além de outros propágulos constituídos de fragmentos de hifas e raízes de $B$. decumbens colonizadas, o quais que resultaram em um número mais provável (NMP) de propágulos infectivos (AN et al., 1990) de pelo menos $2,910^{6}$ unidades por $\mathrm{mL}$. O experimento com fECTOs foi inoculado através de 4 discos de $10 \mathrm{~mm}$ de diâmetro do meio de cultivo Merlin-Norkans modificado (BRUNDRETT et al., 1996), contendo colônias dos isolados de fungos ectomicorrízicos. Os 4 discos foram transferidos para os orifícios ao redor das sementes, conforme Brundrett et al. (1996). Ambos experimentos foram também inoculados com a mistura de estirpes de bactérias fixadoras de nitrogênio provenientes da CCBD (Coleção de Culturas de Bactérias Diazotróficas da Embrapa Agrobiologia) sendo elas as estirpes BR3609 (= SEMIA 6387) de Bradyrhizobium elkanii (GenBank accession number: AY904778) e BR 3617 (= SEMIA 6420) de Bradyrhizobium japonicum (GenBank accession number: AY904786), ambas recomendadas para Acacia mangium. Foram aplicadas ao orifício de plantio por pipetagem de $1 \mathrm{~mL}$ do meio 79 semi-sólido contendo no mínimo $10^{8}$ Unidades Formadoras de Colônia das duas estirpes.

Após a completa germinação das sementes, os experimentos foram desbastados mantendo-se apenas uma planta por recipiente. $\mathrm{O}$ experimento de seleção de FMAs foi conduzido por 120 dias e o experimento de seleção de fECTOs foi conduzido por 150 dias devido ao menor crescimento das plantas neste último. O crescimento vegetativo das plantas foi acompanhado pela avaliação mensal da altura e diâmetro do colo. No final do tempo de condução de ambos os experimentos as plantas foram colhidas e separadas em parte aérea e raízes. A parte aérea foi passada em um medidor de área foliar (LI-COR MODEL 3100 AREA METER), sendo em seguida seca em estufa de circulação de ar a $68{ }^{\circ} \mathrm{C}$ para a tomada de sua massa seca. As raízes foram lavadas em água corrente e tomado seu peso fresco. Foram tomadas de cada planta $1 \mathrm{~g}$ de raízes frescas para a avaliação da colonização micorrízica. O restante das raízes foi seco em estufa a $68^{\circ} \mathrm{C}$ para a quantificação da massa seca.

Para colonização micorrízica, as amostras de raízes dos experimentos foram clarificadas e coradas segundo Koske e Gemma (1989) e Grace e Stribley (1991). No experimento de FMAs a colonização micorrízica foi estimada conforme McGonigle et al. (1990) em microscópio óptico (200x). No experimento com fECTOs a colonização foi estimada pela técnica das intersecções de Giovannetti e Mosse (1980), modificada por Brundrett et al. (1996).

No experimento com FMAs foi ainda quantificado o número dos esporos desses fungos em uma amostra de $50 \mathrm{~cm}^{3}$ de solo. Os esporos foram separados do solo pela associação dos métodos de decantação e peneiramento úmido (GERDEMANN; NICOLSON, 1963) e de centrifugação e flutuação em sacarose (JENKINS, 1964). Posteriormente foi realizada contagem dos esporos em placas de acrílico com anéis concêntricos sob microscópio estereoscópico (40x).

Em ambos os experimentos pela massa da parte aérea seca (MPAS) foram calculadas a eficiência simbiótica dos isolados inoculados (ES). A ES foi estimada pela expressão 


$$
\text { Eficiência simbiótica }(\%)=\frac{(\text { MPAS do tratamento inoculado }- \text { MPAS do tratamento não inoculado })}{\text { MPAS do tratamento não inoculado }} \times 100
$$

Para análise estatística dos dados, foi feita a avaliação de sua normalidade (teste de Lilliefors) e homogeneidade das variâncias dos erros (teste de Cochran e Barttlet) utilizando o programa Sistema de Análises Estatísticas e Genéticas - SAEG versão 5.0 (EUCLYDES, 2007). Posteriormente, os dados normais e homogêneos foram submetidos à análise de variância e as médias foram comparadas pelo teste de Scott Knott 5\% utilizando o programa Sistema de Análise de Variância - SISVAR versão 5.0 (FERREIRA, 2008).

\section{Resultados}

Experimento 1 - Seleção de FMAs

Apenas 30 dias após o plantio e inoculação já havia efeito das diferentes espécies de FMAs na altura da Acacia mangium, sendo que Acaulospora morrowiae $(6,0 \mathrm{~cm})$, Scutellospora calospora $(6,3 \mathrm{~cm})$ e Scutellospora heterogama $(6,2 \mathrm{~cm})$ promoviam maior altura que os demais tratamentos (média $=3,9 \mathrm{~cm}$ ). Aos 60 dias após o plantio, se junta aos três fungos mencionados a espécie Scutellospora gilmorei, todos os quatro proporcionando maior altura de $A$. mangium (média $=16,4 \mathrm{~cm}$ ) que os demais fungos inoculados (média $=7,2 \mathrm{~cm}$ ). O diâmetro do colo aos 60 dias após o plantio corrobora os dados de altura, sendo que as mesmas quatro espécies (média $=2,1 \mathrm{~mm}$ ) apresentavam maior diâmetro que os demais tratamentos (média $=1,1 \mathrm{~mm}$ ).

Aos 120 dias após o plantio os fungos Acaulospora morrowiae, Entrophospora contigua, Gigaspora margarita, Scutellospora calospora, Scutellospora gilmorei e Scutellospora heterogama promoviam maior altura que os demais tratamentos e não diferiam entre si (Tabela 2). Formando um segundo grupo, ficaram as espécies Entrophospora colombiana e Glomus clarum que promoviam ainda maior altura que a testemunha não inoculada. Somente foram iguais a testemunha não inoculada as plantas com Acaulospora laevis e Scutellospora pellucida. Praticamente o mesmo resultado foi observado para o diâmetro do colo aos 120 dias, excetuando-se Gigaspora margarita que saiu do grupo das que mais promoveram o crescimento para o segundo grupo (Tabela 2).

Desta forma, a inoculação de $A$. morrowiae, $S$. calospora, S. heterogama, S. gilmorei promoveu crescimento inicial acelerado da $A$. mangium entre 30 e 60 dias após o plantio, sendo o incremento médio na altura superior a $10 \mathrm{~cm}$. Após este crescimento acelerado, possivelmente já devido a uma limitação das raízes no recipiente, a velocidade de crescimento das plantas com estes fungos diminuiu, passando a ser em média $6 \mathrm{~cm}$ entre 60 e 90 dias e $5 \mathrm{~cm}$ entre 90 e 120 dias. Com isso, aos 120 dias após o plantio outros fungos promoviam altura igual aos quatro primeiros fungos a se destacarem.

Em relação a produção de massa seca da parte aérea (Tabela 2) destacaram-se as plantas de $A$. mangium inoculadas com $A$. morrowiae, S. calospora e $S$. gilmorei que apresentaram maior massa que as inoculadas com os demais tratamentos. Plantas com E. contigua, G. margarita e S. heterogama apresentaram massa superior ao do tratamento não inoculado. Plantas com A. laevis, E. colombiana, $G$. clarum e $S$. pellucida não diferiram da testemunha não inoculada. 
Tabela 2. Altura de plantas, diâmetro do colo, massa da parte aérea seca (MPAS), massa das raízes secas (MRS) e área foliar de Acacia mangium inoculadas com diferentes espécies de fungos micorrízicos arbusculares (FMAs) aos 120 dias após plantio em casa de vegetação.

\begin{tabular}{lrrrrrr}
\hline Tratamentos de inoculação & $\begin{array}{c}\text { Altura } \\
(\mathrm{cm})\end{array}$ & $\begin{array}{c}\text { Diâmetro do } \\
\text { colo }(\mathrm{mm})\end{array}$ & MPAS $(\mathrm{g})$ & MRS $(\mathrm{g})$ & $\begin{array}{c}\text { Área Foliar } \\
\left(\mathrm{cm}^{2}\right)\end{array}$ \\
\hline Acaulospora laevis & $10,08 \mathrm{c}$ & $1,42 \mathrm{c}$ & $0,38 \mathrm{c}$ & $0,50 \mathrm{~d}$ & $31 \mathrm{c}$ \\
Acaulospora morrowiae & $27,10 \mathrm{a}$ & $3,22 \mathrm{a}$ & $2,84 \mathrm{a}$ & $2,87 \mathrm{~b}$ & $200 \mathrm{a}$ \\
Entrophospora colombiana & $15,78 \mathrm{~b}$ & $2,10 \mathrm{~b}$ & $0,72 \mathrm{c}$ & $1,42 \mathrm{c}$ & $98 \mathrm{~b}$ \\
Entrophospora contigua & $27,40 \mathrm{a}$ & $3,32 \mathrm{a}$ & $2,02 \mathrm{~b}$ & $2,56 \mathrm{~b}$ & $113 \mathrm{~b}$ \\
Gigaspora margarita & $25,18 \mathrm{a}$ & $2,96 \mathrm{~b}$ & $2,00 \mathrm{~b}$ & $2,19 \mathrm{~b}$ & $116 \mathrm{~b}$ \\
Glomus clarum & $13,20 \mathrm{~b}$ & $1,70 \mathrm{~b}$ & $0,52 \mathrm{c}$ & $0,90 \mathrm{c}$ & $90 \mathrm{~b}$ \\
Scutellospora calospora & $27,90 \mathrm{a}$ & $3,42 \mathrm{a}$ & $3,20 \mathrm{a}$ & $3,32 \mathrm{a}$ & $202 \mathrm{a}$ \\
Scutellospora gilmorei & $28,84 \mathrm{a}$ & $3,52 \mathrm{a}$ & $3,05 \mathrm{a}$ & $3,53 \mathrm{a}$ & $189 \mathrm{a}$ \\
Scutellospora heterogama & $22,60 \mathrm{a}$ & $3,10 \mathrm{a}$ & $2,34 \mathrm{~b}$ & $2,61 \mathrm{~b}$ & $120 \mathrm{~b}$ \\
Scutellospora pellucida & $9,12 \mathrm{c}$ & $1,32 \mathrm{c}$ & $0,33 \mathrm{c}$ & $0,53 \mathrm{~d}$ & $20 \mathrm{c}$ \\
Testemunha não inoculada & $6,30 \mathrm{c}$ & $1,12 \mathrm{c}$ & $0,07 \mathrm{c}$ & $0,19 \mathrm{~d}$ & $10 \mathrm{c}$ \\
\hline C.V. ${ }^{(1)}$ (\%) & 23,9 & 18,8 & 27,6 & 29,5 & 20,2 \\
\hline
\end{tabular}

Médias seguidas de mesma letra na coluna, não diferem entre si pelo teste de Scott Knott a 5\%.

(1): Coeficiente de variação (C.V.) da análise de variância;

Fonte: Elaboração dos autores.

Para a massa das raízes secas (Tabela 2), o comportamento foi semelhante ao da parte aérea, porém mais grupos foram discriminados. Plantas inoculadas com $S$. calospora e $S$. gilmorei apresentaram maior massa radicular que os demais tratamentos. Em seguida agruparam-se as plantas inoculadas com A. morrowiae, E. contigua, $G$. margarita e $S$. heterogama. Inferiores a este grupo, mas ainda maiores que a testemunha não inoculada ficou o grupo das plantas inoculadas com E. colombiana e G. clarum. Não diferiram da testemunha não inoculada as plantas inoculadas com A. laevis e S. pellucida.

A área foliar das plantas mostra mais claramente a diferença de crescimento entre os tratamentos de inoculação (Tabela 2). Os fungos A. morrowiae, $S$. calospora e $S$. gilmorei proporcionaram maior área foliar nas plantas. Mudas inoculadas com $E$. colombiana, E. contigua, G. margarita, G. clarum e $S$. heterogama apresentam área foliar com superfície intermediária, mas superior a testemunha. Os fungos A. laevis e $S$. pellucida proporcionaram menor área foliar, não diferindo da testemunha não inoculada, apesar de terem mais que o dobro da área foliar deste controle experimental.

A colonização micorrízica apresentou grande variação entre as diferentes espécies de FMAs testadas (Tabela 3). Valores elevados de colonização radicular (acima de 80\%) foram encontrados em plantas inoculadas com S. heterogama e E. colombiana, que promoveram colonização superior às demais espécies inoculadas. S. gilmorei, $A$. morrowiae e $S$. calospora também promoveram colonização bastante elevada, acima de 70\%, formando um segundo escalão. Em seguida, um grupo formado apenas por G. margarita com 64\% de colonização. Um quarto grupo formado por $G$. clarum e E. contigua apresentaram valores medianos de colonização (49\% e 55\%, respectivamente). $\mathrm{Na}$ sequência, outro grupo formado apenas por $S$. pellucida (39\% de colonização) e o último grupo formado apenas por A. laevis (22\%). No tratamento não inoculado não foi observada colonização micorrízica, indicando a ausência de contaminação dos vasos que compunham este tratamento. 
Tabela 3. Colonização micorrízica das raízes, número de esporos na rizosfera, número e massa de nódulos secos nas raízes de Acacia mangium inoculadas com diferentes espécies de fungos micorrízicos arbusculares (FMAs) aos 120 dias após plantio em casa de vegetação.

\begin{tabular}{|c|c|c|c|c|}
\hline Tratamentos de inoculação & $\begin{array}{c}\text { Colonização } \\
\text { micorrízica }\end{array}$ & $\begin{array}{l}\text { Número de } \\
\text { esporos }\end{array}$ & $\begin{array}{l}\text { Número de } \\
\text { nódulos* }\end{array}$ & $\begin{array}{l}\text { Massa de nódulos } \\
\text { secos* }\end{array}$ \\
\hline & $\%$ & $\begin{array}{l}\mathrm{N}^{0} .50 \mathrm{~cm}^{-3} \mathrm{de} \\
\quad \text { solo seco }\end{array}$ & $\mathrm{N}^{\mathrm{o}}$. planta $^{-1}$ & g $\cdot$ planta $^{-1}$ \\
\hline Acaulospora laevis & $22 \mathrm{f}$ & $214 \mathrm{c}$ & $0,0 \mathrm{~b}$ & $0,00 \mathrm{~b}$ \\
\hline Acaulospora morrowiae & $74 \mathrm{~b}$ & $411 \mathrm{a}$ & 50,6 a & 0,03 a \\
\hline Entrophospora colombiana & $84 \mathrm{a}$ & $349 \mathrm{~b}$ & $0,0 \mathrm{~b}$ & $0,00 \mathrm{~b}$ \\
\hline Entrophospora contigua & $49 \mathrm{~d}$ & $336 \mathrm{~b}$ & $12,0 \mathrm{~b}$ & $0,01 \mathrm{~b}$ \\
\hline Gigaspora margarita & $64 \mathrm{c}$ & $218 \mathrm{c}$ & 32,2 a & 0,03 a \\
\hline Glomus clarum & $55 \mathrm{~d}$ & 383 a & $1,4 \mathrm{~b}$ & $0,00 \mathrm{~b}$ \\
\hline Scutellospora calospora & $73 \mathrm{~b}$ & $226 \mathrm{c}$ & 34,0 a & 0,03 a \\
\hline Scutellospora gilmorei & $79 \mathrm{~b}$ & $297 \mathrm{~b}$ & 33,8 a & 0,02 a \\
\hline Scutellospora heterogama & 89 a & $360 \mathrm{~b}$ & 35,4 a & 0,02 a \\
\hline Scutellospora pellucida & 39 e & $169 \mathrm{c}$ & $0,0 \mathrm{~b}$ & $0,00 \mathrm{~b}$ \\
\hline Controle Não Inoculado & $0 \mathrm{~g}$ & $0 \mathrm{~d}$ & $0,0 \mathrm{~b}$ & $0,00 \mathrm{~b}$ \\
\hline C.V. ${ }^{(1)}(\%)$ & 10,8 & 14,9 & 61,4 & 0,84 \\
\hline
\end{tabular}

Médias seguidas de mesma letra na coluna, não diferem entre si pelo teste de Scott Knott a 5\%.

(1): Coeficiente de variação (C.V.) da análise de variância.

*: Dados transformados pela equação: $\mathrm{y}=$ raiz quadrada de $\mathrm{x}+0,5$; apresentados destransformados.

Fonte: Elaboração dos autores.

Quanto a esporulação dos FMAs na rizosfera verificou-se que $A$. morrowiae e $G$. clarum foram os que mais esporularam, em média 397 esporos em 50 $\mathrm{cm}^{3}$ de solo (Tabela 3). Os fungos E. colombiana, $E$. contigua, S. gilmorei e $S$. heterogama, apresentaram em média 335 esporos em $50 \mathrm{~cm}^{3}$ de solo formando um segundo grupo. Por fim, A. laevis, G. margarita, $S$. calospora e $S$. pellucida formam o grupo com menor esporulação na rizosfera sendo em média 207 esporos em $50 \mathrm{~g}$ de solo. Não se observou esporulação no tratamento não inoculado. Excluindo-se este controle, não houve correlação da esporulação com a colonização micorrízica $(\mathrm{r}=$ $0,52)$ e com a massa da parte aérea seca $(r=0,21)$.

Anodulação por bactérias fixadoras de nitrogênio atmosférico em $A$. mangium não foi encontrada em todos os tratamentos, embora todos tenham sido inoculados (Tabela 3). As plantas inoculadas com $A$. morrowiae, G. margarita, S. calospora, S. gilmorei e $S$. heterogama apresentaram maior número e peso de nódulos. Os demais tratamentos não diferiram a nodulação das raízes do tratamento não inoculado, o qual não apresentou nodulação. Houve correlação entre o número e peso de nódulos com a massa da parte aérea seca $(\mathrm{r}=0,91)$.

A estimativa da eficiência simbiótica de cada tratamento de inoculação é apresentada na figura 1. Os fungos A. morrowiae, S. calospora e S. gilmorei foram os mais eficientes em promover a massa da parte aérea seca de $A$. mangium proporcionando um incremento em torno de $4000 \%$ maior que a planta não inoculada. E. contigua, G. margarita e $S$. heterogama também apresentaram grande eficiência simbiótica promovendo o crescimento da planta em torno de $3000 \%$ superior ao da planta não inoculada. Os fungos E. colombiana e G. clarum apresentaram eficiência simbiótica moderada, promovendo 900 e $600 \%$, respectivamente o crescimento da planta em relação à não inoculada. Os fungos $A$. laevis e $S$. pellucida apresentaram baixa eficiência simbiótica comparados aos demais fungos, promovendo um crescimento de 430 e $350 \%$, respectivamente, superior ao da testemunha não inoculada. 
Figura 1. Eficiência simbiótica (\%) de diferentes espécies de fungos micorrízicos arbusculares (FMAs) inoculados em Acacia mangium. A. lae: Acaulospora laevis; A. mor: Acaulospora morrowiae; E. col: Entrophospora colombiana; E. con: Entrophospora contigua; G. mar: Gigaspora margarita; G. cla: Glomus clarum; S. cal: Scutellospora calospora; S.gil: Scutellospora gilmorei; S. het: Scutellospora heterogama; S. pel: Scutellospora pellucida.

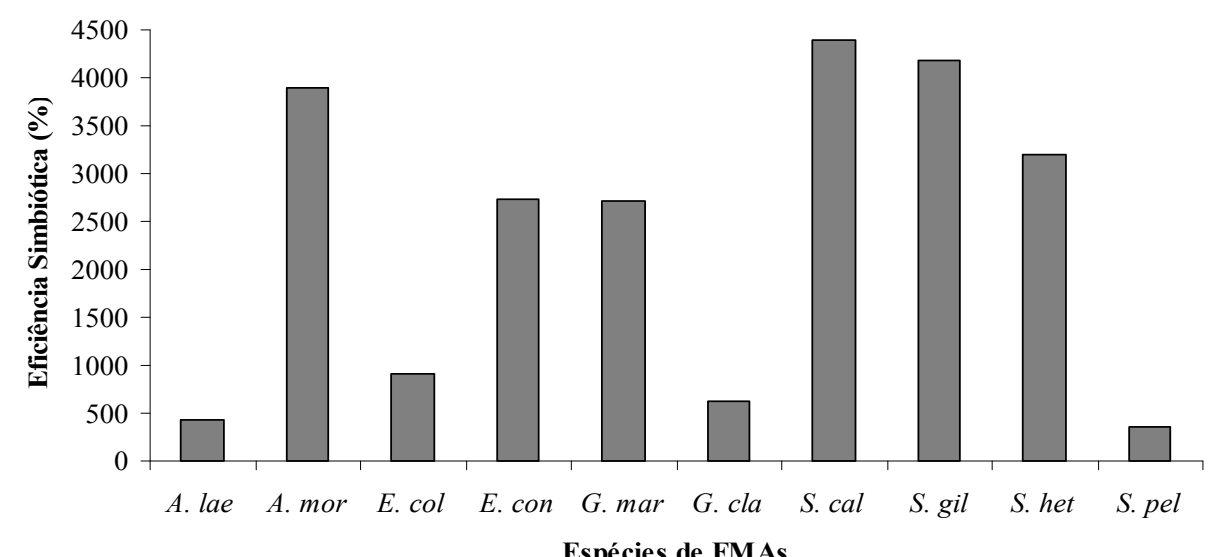

Fonte: Elaboração dos autores.

\section{Experimento 2 - Seleção de fECTOs}

A inoculação dos isolados de fungos ectomicorrízicos não promoveu efeito significativo no crescimento de $A$. mangium. Não houve diferenças entre os tratamentos tanto para a altura das plantas quanto para o diâmetro do colo avaliados durante o período de condução do experimento. A altura de plantas, diâmetro do colo, massa da parte aérea seca, massa das raízes secas e área foliar avaliados por ocasião da colheita do experimento também não apresentaram diferenças entre os tratamentos de inoculação (Tabela 4). A eficiência simbiótica de todos os isolados testados foi baixa ou mesmo inexistente.

Tabela 4. Altura de plantas, diâmetro do colo, massa da parte aérea seca (MPAS), massa das raízes secas (MRS) e área foliar de Acacia mangium inoculadas com diferentes isolados de fungos ectomicorrízicos (fECTOs) aos 150 dias após plantio em casa de vegetação.

\begin{tabular}{lccccc}
\hline Tratamentos de inoculação & $\begin{array}{c}\text { Altura } \\
(\mathrm{cm})\end{array}$ & $\begin{array}{c}\text { Diâmetro do } \\
\text { colo }(\mathrm{mm})\end{array}$ & $\begin{array}{c}\text { MPAS* } \\
(\mathrm{g})\end{array}$ & $\begin{array}{c}\text { MRS* } \\
(\mathrm{g})\end{array}$ & $\begin{array}{c}\text { Área Foliar* } \\
\left(\mathrm{cm}^{2}\right)\end{array}$ \\
\hline UFSC Pt 116 & $6,70 \mathrm{a}$ & $1,17 \mathrm{a}$ & $0,16 \mathrm{a}$ & $0,19 \mathrm{a}$ & $3,10 \mathrm{a}$ \\
UFSC Pt 24 & $5,44 \mathrm{a}$ & $0,97 \mathrm{a}$ & $0,15 \mathrm{a}$ & $0,15 \mathrm{a}$ & $2,65 \mathrm{a}$ \\
UFSC Pt 193 & $7,76 \mathrm{a}$ & $1,10 \mathrm{a}$ & $0,49 \mathrm{a}$ & $0,32 \mathrm{a}$ & $5,53 \mathrm{a}$ \\
UFSC Pt 187 & $7,80 \mathrm{a}$ & $1,20 \mathrm{a}$ & $0,41 \mathrm{a}$ & $0,14 \mathrm{a}$ & $6,60 \mathrm{a}$ \\
O 64-ITA 6 & $8,25 \mathrm{a}$ & $0,95 \mathrm{a}$ & $0,27 \mathrm{a}$ & $0,28 \mathrm{a}$ & $4,00 \mathrm{a}$ \\
O 40-ORS 7870 & $6,60 \mathrm{a}$ & $1,00 \mathrm{a}$ & $0,15 \mathrm{a}$ & $0,24 \mathrm{a}$ & $3,10 \mathrm{a}$ \\
Controle Não Inoculado & $7,30 \mathrm{a}$ & $1,20 \mathrm{a}$ & $0,47 \mathrm{a}$ & $0,22 \mathrm{a}$ & $2,80 \mathrm{a}$ \\
\hline C.V. ${ }^{(1)}$ (\%) & 32,00 & 30,65 & 20,70 & 7,46 & 22,6 \\
\hline
\end{tabular}

Médias seguidas de mesma letra na coluna, não diferem entre si pelo teste de Scott Knott a 5\%.

(1): Coeficiente de variação (C.V.) da análise de variância.

*: Dados transformados pela equação: $\mathrm{y}=$ raiz quadrada de $\mathrm{x}+0,5$; apresentados destransformados.

Fonte: Elaboração dos autores. 
Apesar da ausência de resposta das plantas aos fECTOs inoculados foram observadas alguma taxa de colonização micorrízica nas raízes de $A$. mangium (Figura 2). Verificou-se que o isolado O 64-ITA 6 apresentou a maior colonização micorrízica (33\%) seguido dos isolados UFSC Pt 193 e O 40-ORS 7870. Os demais isolados testados não colonizaram as raízes de $A$. mangium, assim como a testemunha não inoculada. Não foram verificados nódulos de bactérias fixadoras de nitrogênio neste experimento apesar da inoculação destas.

Figura 2. Colonização micorrízica em raízes de Acacia mangium inoculada com diferentes isolados de fungo ectomicorrízico (fECTO) aos 150 dias após plantio em casa de vegetação e em plantas não inoculadas (N.I.). Barras com mesmas letras, não diferem entre si pelo teste de Scott Knott a 5\%. Coeficiente de variação da análise de variância: $30,2 \%$.

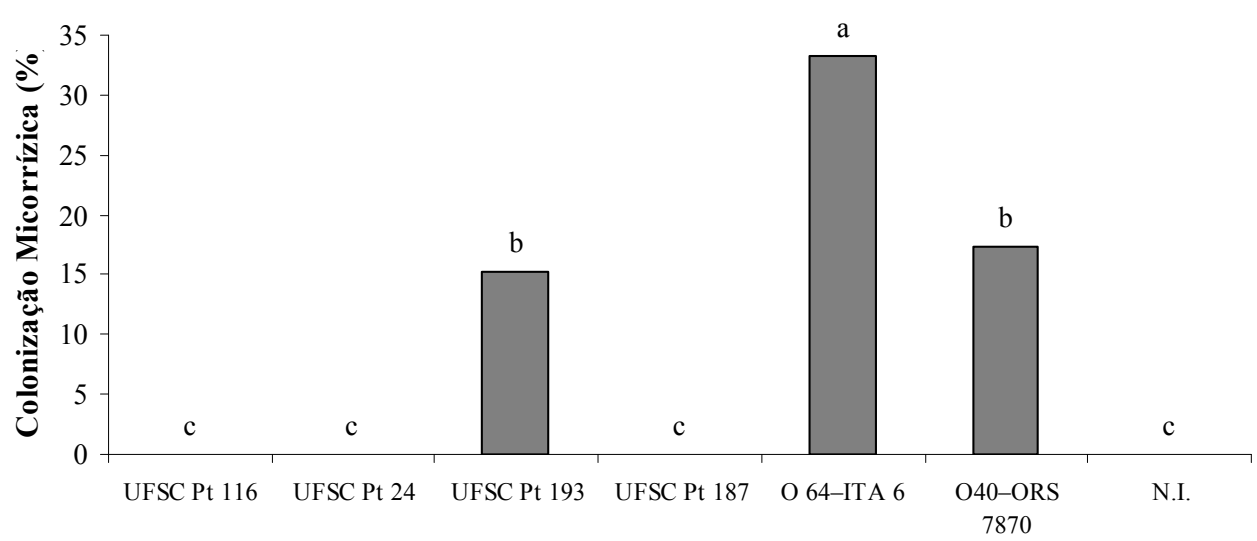

Isolados de fECTOs

Fonte: Elaboração dos autores.

\section{Discussão}

A verificação de que todas as espécies de FMAs estudadas promoveram algum crescimento da planta e que todas promoveram colonização radicular fornece forte indicativo de que $A$. mangium apresenta alto grau de dependência micorrízica (SIQUEIRA; SAGGIN JÚNIOR, 2001; JANOS, 2007). Neste caso, a diferença de crescimento observada entre os tratamentos de espécies fúngicas deve-se à variação da responsividade (SIQUEIRA; SAGGIN JÚNIOR, 2001; JANOS, 2007), a qual é grandemente influenciada pela eficiência de cada combinação fungo-planta-ambiente (SMITH; SMITH; JAKOBSEN, 2003). Conforme descrito no material e métodos, as condições edáficas, de casa de vegetação e de planta hospedeira foram padronizadas entre os tratamentos, de modo que a variação da responsividade observada pode ser atribuída à variabilidade de cada tratamento/ genótipo fúngico.

Desta forma, os fungos mais eficientes em promover o crescimento de A. mangium foram A. morrowiae, S. calospora e S. gilmorei. Todos os três apresentaram colonização micorrízica na faixa de $70 \%$, o que aparentemente forneceu um equilíbrio ideal para Acacia mangium entre benefício micorrízico e o dreno de fotossintatos promovido pelos fungos (LI et al., 2008). Fungos que promoveram colonizações acima de $80 \%$ ( $E$. colombiana e $S$. heterogama) não apresentaram eficiência máxima em promover o crescimento de A. mangium, assim como os que apresentaram colonizações abaixo de 70\%. Colonizações micorrízicas abaixo de $40 \%$ mostraram-se ineficazes 
em promover o crescimento de A. mangium em relação à testemunha não inoculada, apesar de que para outras espécies arbóreas esse nível de colonização seja considerado bom (ZANGARO FILHO et al., 2000). Acacia mangium mostra grande variação na colonização micorrízica (2178\%) em diferentes estudos (MARTIN-LAURENT et al., 1999; FOUNOUNE et al., 2002; GHOSH; VERMA, 2006; AGGANGAN; MOON; HAN, 2010; JEYANNY; LEE; RASIDAH, 2011) variando com o solo, fertilização, condição de cultivo e microbiota acompanhante. No presente estudo, onde houve variação apenas da espécie de fungo inoculado, a variação da colonização se deve à fisiologia da relação entre os diferentes FMAs e $A$. mangium (KOLTAI; KAPULNIK, 2010). Tanto a disponibilidade de $\mathrm{P}$ no solo quanto à carga genética de dependência micorrízica que a planta possui estão envolvidos nos mecanismos de sinalização e controle da colonização radicular (KIRIACHEK et al., 2009).

Não houve relação da esporulação na rizosfera com a colonização radicular e a produção de massa seca pelas plantas, confirmando-se um fato frequente na literatura de que a esporulação dos FMAs é pouco afetada pela colonização micorrízica e tem pouca relação com a eficiência do fungo em promover crescimento (AGUÍN et al., 2004; CAVALCANTE et al., 2002). Portanto, a quantidade de esporos observada nos diferentes tratamentos do presente estudo implica em diferentes estratégias de sobrevivência das diferentes espécies de FMAs (OEHL et al., 2009).

A formação de nódulo de bactérias fixadoras de nitrogênio ocorreu nos tratamentos fúngicos que promoveram colonização micorrízica superior a $49 \%$, sendo a única excessão as plantas inoculadas com E. colombiana que apresentou $84 \%$ de colonização, mas não apresentou nodulação. A observação de correlação entre a nodulação e a produção de massa seca das plantas reforça o papel dos FMAs em promover a associação de bactérias fixadoras de nitrogênio com a planta hospedeira. Portanto, o presente trabalho corrobora a relação das micorrizas com a fixação biológica de nitrogênio, onde os FMAs tem papel relevante na promoção da associação bactéria x planta (JESUS; SCHIAVO; FARIA, 2005). Este efeito já bem conhecido possivelmente está relacionado com o papel dos FMAs em fornecer P para as leguminosas, já que o processo de fixação biológica de nitrogênio é altamente exigente em ATP (SUBRAMANIAN et al., 2011).

A eficiência simbiótica dos tratamentos fúngicos em A. mangium foi de magnitude muito alta, sendo acima de $350 \%$ e chegando a $4000 \%$ nos fungos mais eficientes. Estes resultados indicam a variação na responsividade de $A$. mangium aos diferentes fungos por estes promoverem simbioses com diferentes habilidades, funções e principalmente capacidade de absorção de fósforo (JANOS, 2007). A diferença das combinações fungo x planta em absorver fósforo foi evidenciada no estudo de Smith, Smith e Jakobsen (2003). Esses autores demonstraram que as combinações fungo x planta promovem grandes diferenças na contribuição relativa de absorção de $\mathrm{P}$ via raízes ou via hifas. As combinações mais eficientes fornecem 100\% do $\mathrm{P}$ absorvido via hifa, tornando insignificante a absorção de $\mathrm{P}$ via raízes. Os autores também observam que fungos com capacidades similares de absorção de $\mathrm{P}$ via hifa diferem na eficiência, neste caso possivelmente por diferença do fungo na requisição de carbono orgânico da planta. Portanto, a diversidade funcional nas combinações fungo-planta possivelmente esta ligada às proteínas transportadoras de $\mathrm{Pi}$ e fotossintatos nas paredes celulares da interface fungo-planta. O controle deste transporte está ligado à genética de ambos os simbiontes (BUCHER, 2007). Para aumentar o conhecimento sobre as combinações fungo-planta eficientes são necessários estudos elucidando os sinais bioquímicos que controlam a expressão dos genes das proteínas transportadoras na simbiose micorrízica. 
Em relação à ausência de respostas de $A$. mangium a inoculação dos fECTOs, este foi um comportamento ligado à característica dos fungos ectomicorrízicas serem bastante específicos nas combinações com suas planta hospedeira (BRUNDRETT, 2002; BRUNS; BIDARTONDO; TAYLOR, 2002). Esta simbiose caracteriza-se pela baixa diversidade de plantas hospedeiras, porém com alta diversidade fúngica e alta especificidade dos isolados fúngicos (MOLINA; MASSICOTTE; TRAPPE, 1992). Apesar disto houve colonização das raízes por três isolados de fECTOs estudados, o que sugere a possibilidade de ter havido também pouca adaptação dos isolados fúngicos ao solo utilizado. Como houve colonização, os isolados com maior nível de colonização podem ser selecionados por esta característica para estudos futuros com $A$. mangium em outras condições ambientais.

A inexistência de isolados de fECTOs eficientes e a baixa colonização verificada no presente estudo também pode estar ligada a associação ectomicorrízica poder ocorrer estádios diferenciados do crescimento da planta (LAST; DIGHTON; MASON, 1987). Existem isolados fúngicos que se associam a plantas jovens e aqueles que se associam as plantas maduras, e ainda aqueles que se associam a plantas em várias idades (MASON, 1982; LAST; DIGHTON; MASON, 1987). Assim, estudos mais prolongados podem ser feitos com $A$. mangium e os isolados de fECTOs que colonizaram para avaliar a possibilidade de efeito tardio desta simbiose.

Outro aspecto a ser considerado é que vários estudos mostram que a inoculação de espécies florestais com fECTOs proporcionam incremento menor que a inoculação por FMAs. O incremento com fECTOS raramente chegam a 100\% em relação ao controle não inoculado (FOUNOUNE et al., 2002; JAYAKUMAR; TAN, 2005; AGGANGAN; MOON; HAN, 2010). É importante considerar que a formação, manutenção e funcionamento das estruturas fúngicas das ectomicorrizas pode ter um maior gasto de fotossintatos que a dos FMAs.
A alocação de carbono para fECTOs é relatado chegar a 22\% (HOBBIE, 2006) enquanto que para FMAs ficam abaixo de 16\% (KASCHUK et al., 2009). Além do dreno de fotossintatos, o impacto da simbiose no crescimento da planta depende da fertilidade do solo, de outros fatores ambientais, da idade ou fase de desenvolvimento da planta e, em particular, da capacidade do fungo de compensar e superar o gasto fotossintético com benefícios à planta que envolve absorção de nutrientes e água e resistência a estresses bióticos e abióticos (RONSHEIM, 2012).

\section{Conclusões}

Os Isolados de fungos ectomicorrízicos UFSC Pt193, O 64-ITA6 e, O 40-ORS 7870 colonizaram as raízes de Acacia mangium, mas não foram eficientes em promover o crescimento desta espécie arbórea.

Os fungos micorrízicos arbusculares promovem maior crescimento de Acacia mangium que os fungos ectomicorrízicos, sendo as espécies Scutellospora calospora,Scutellospora heterogama, Scutellospora gilmorei e Acaulospora morrowiae as que promovem simbioses mais eficientes.

Mudas de Acacia mangium são muito responsivas a inoculação de FMAs e dependem de elevada colonização micorrízica, entre 40 e $80 \%$, para obter benefícios relevantes da simbiose no crescimento e nodulação.

Os FMAs eficientes tem papel relevante na promoção da associação entre bactérias fixadoras de nitrogênio e Acacia mangium.

\section{Agradecimentos}

Os autores agradecem o apoio da Embrapa Agrobiologia, Curso de Pós-Graduação em Agronomia - Ciência do Solo (CPGA-CS) e da Coordenação de Aperfeiçoamento de Pessoal de Nível Superior (CAPES). 


\section{Referências}

AGGANGAN, N. S.; MOON, H. K.; HAN, S. H. Growth response of Acacia mangium Willd. seedlings to arbuscular mycorrhizal fungi and four isolates of the ectomycorrhizal fungus Pisolithus tinctorius (Pers.) Coker and Couch. New Forests, New York, v. 39, n. 2, p. 215-230, 2010.

AGUÍN, O.; MANSILlA, J. P.; VILARIÑO, A.; SAINZ, M. J. Effects of mycorrhizal inoculation on root morphology and nursery production of three grapevine rootstocks. American Journal of Enology and Viticulture, Davis, v. 55, n. 1, p. 108-111, 2004.

AN, Z. Q.; HENDRIX, J. W.; HERSHMAN, D. E.; HENSON, G. T. Evaluation of the "most probable number" (MPN) and wet-sieving methods for determining soil-borne population of endogonaceous mycorrhizal fungi. Mycologia, New York, v. 82, n. 5, p. 576-581, 1990.

BRUNDRETT, M. C. Coevolution of roots and mycorrhizas of land plants. New Phytologist, Lancaster, v. 154, n. 2, p. 275-304, 2002.

BRUNDRETT, M.; BOUGHER, N.; DELL, B.; GROVE, T; MALAJCZUK, N. (Ed.). Working with mycorrhizas in forestry and agriculture. Canberra: Australian Centre For International Agricultural Research, 1996. 374 p.

BRUNS, T. D.; BIDARTONDO, M. I.; TAYLOR, D. L. Host Specificity in ectomycorrhizal communities: what do the exceptions tell us? Integrative and Comparative Biology, Oxford, v. 42, n. 2, p. 352-359, 2002.

BUCHER, M. Functional biology of plant phosphate uptake at root and mycorrhiza interfaces. New Phytologist, Lancaster, v. 173, n. 1, p. 11-26, 2007.

CAVAlCANTE, U. M. T.; MAIA, L. C.; MELO, A. M. M.; SANTOS, V. F. Influência da densidade de fungos micorrízicos arbusculares na produção de maracujazeiroamarelo. Pesquisa Agropecuária Brasileira, Brasília, v. 37, n. 5, p. 643-649, 2002.

EUCLYDES, R. F. SAEG-sistema para análises estatísticas, versão 9.1. Viçosa: Fundação Arthur Bernardes, Universidade Federal de Viçosa/UFV/DBG, 2007.

FOOD AND AGRICULTURE ORGANIZATION FAO. 2006. World reference base for soil resources. World soil resources reports, 103. Roma, 2006.

FERREIRA, D. F. SISVAR: um programa para análises e ensino de estatística. Revista Symposium, Lavras, v. 6, n. 1, p. 36-41, 2008.
FOUNOUNE, H.; DUPONNOIS, R.; BÂ, A. M.; BOUAMI, F. E. Influence of the dual arbuscular endomycorrhizal / ectomycorrhizal symbiosis on the growth of Acacia holosericea (A. Cunn. ex G. Don) in glasshouse conditions. Annals of Forest Science, Les Ulis, v. 59, n. 1, p. 93-98, 2002.

FRANCO, A. A.; DIAS, L. E.; FARIA, S. M.; CAMPELlO, E. F. C.; SILVA, E. M. R. da. Uso de leguminosas florestais noduladas e micorrizadas como agentes de recuperação e manutenção da vida do solo: um modelo tecnológico. In: ESTEVES, F. A. (Ed.). Oecologia Brasiliensis. Estrutura, funcionamento e manejo de ecossistemas brasileiros. Rio de Janeiro: Instituto de Biologia/UFRJ, 1995. v. 1, p. 459-467.

GERDEMANN, J. W.; NICOLSON, T. H. Spores of mycorrhizal Endogone species extracted from soil by wet sieving and decanting. Transaction of British Mycology Society, London, v. 46, n. 2, p. 235-244, 1963.

GHOSH, S.; VERMA, N. K. Growth and mycorrhizal dependency of Acacia mangium willd. inoculated with three vesicular arbuscular mycorrhizal fungi in lateritic soil. New Forests, New York, v. 31, n. 1, p. 75-81, 2006.

GIOVANNETTI, N.; MOSSE, B. An evalution of techniques to measure vesicular-arbuscular infection in roots. The New Phytologist, Oxford, v. 84, n. 4, p. 489$500,1980$.

GRACE, C.; STRIBLEY, D. P. A. A safer procedure for roution staining of vesicular-arbuscular mycorrhizal fungi. Mycological Research, Cambridge, v. 5, n. 10, p. 1160-1162, 1991.

HOBBIE, E. A. Carbon allocation to ectomycorrhizal fungi correlates with belowground allocation in culture studies. Ecology, Davis, v. 87, n. 3, p. 563-569, 2006.

JANOS, D. P. Plant responsiveness to mycorrhizas differs from dependence upon mycorrhizas. Mycorrhiza, Québec, v. 17, n. 2, p. 75-92, 2007.

JAYAKUMAR, P.; TAN, T. K. Growth performance and nodulation response of Acacia mangium co-inoculated with Bradyrhizobium sp. and Pisolithus tinctorius. Journal Symbiosis, Rehovot, v. 40, n. 2, p. 109-114, 2005.

JENKINS, W. R. A rapid centrifugal-flotation technique for separating nematodes from soil. Plant Disease Report, Beltsville, v. 48, n. 1, p. 692, 1964.

JESUS, E. C.; SCHIAVO, J. A.; FARIA, S. M. Dependência de micorrizas para nodulação de leguminosas arbóreas tropicais. Revista Árvore, Viçosa, v. 29, n. 4, p. 545-552, 2005. 
JEYANNY, V.; LEE, S. S.; RASIDAH, K. W. Effects of arbuscular mycorrhizal inoculation and fertilisation on the growth of Acacia mangium seedlings. Journal of Tropical Forest Science, Bahasa, v. 23, n. 4, p. 404-409, 2011.

KASCHUK, G.; KUYPER, T. W.; LEFFELAAR, P. A.; HUNGRIA, M.; GILLER, K. E. Are the rates of photosynthesis stimulated by the carbon sink strength of rhizobial and arbuscular mycorrhizal symbioses? Soil Biology and Biochemistry, Oxford, v. 41, n. 6, p. 12331244, 2009.

KIRIACHEK, S. G.; AZEVEDO, L. C. B. de; PERES, L. E. P.; LAMBAIS, M. R. Regulação do desenvolvimento de micorrizas arbusculares. Revista Brasileira de Ciência do Solo, Viçosa, v. 33, n. 1, p. 1-16, 2009.

KLIRONOMOS, J. N. Host-specificity and functional diversity among arbuscular mycorrhizal fungi. In: BELL, C. R.; BRYLINSKY, M.; JOHNSON-GREEN, P. (Ed.). Microbial biosystems: new frontiers. proceedings of the 8th international symposium on microbial ecology. Halifax, Canada: Atlantic Canada Society for Microbial Ecology, 2000. p. 845-851. Disponível em: <http://plato. acadiau.ca/isme/symposium26/klironomos.pdf $>$. Acesso em: 07 ago. 2012.

KOLTAI, H.; KAPULNIK, Y. (Ed.). Arbuscular mycorrhiza: physiology and function. $2^{\text {nd }}$ ed. Dordrecht, Heidelberg, London, New York: Springer, 2010. 323 p.

KOSKE, R. E.; GEMMA, J. N. A modified procedure for sting roots to detect VA mycorrhizas. Mycological Research, Cambridge, v. 92, n. 4, p. 486-488, 1989.

LAST, F. T.; DIGHTON, J.; MASON, P. A. Successions of sheathing mycorrhizal fungi. Trends in Ecology and Evolution, Oxford, v. 2, n. 6, p. 157-161, 1987.

LI, H.; SMITH, F. A.; DICKSON, S.; HOLLOWAY, R. E.; SMITH, S. E. Plant growth depressions in arbuscular mycorrhizal symbioses: not just caused by carbon drain? New Phytologist, Oxford, v. 178, n. 4, p. 852-862, 2008.

MARTIN-LAURENT, F.; LEE, S. K.; THAM, F. Y.; JIE, H.; DIEM, H. G. Aeroponic production of Acacia mangium saplings inoculated with AM fungi for reforestation in the tropics. Forest Ecology and Management, Netherlands, v. 122, n. 3, p. 199-207, 1999.

MASON, P. A. Ecology of some fungi associated with an ageing stand of birches (Betula pendula and B. pubescens). Forest Ecology and Management, Netherlands, v. 4, n. 1, p. 19-39, 1982.
McGONIGLE, T. P.; MILLER, M. H.; EVANS, D. G.; FAIRCHILD, G. L.; SWAN, J. A. A new method which gives an objective measure of colonization of roots by vesicular-arbuscular mycorrhizal fungi. New Phytologist, Lancaster, v. 115, n. 3, p. 495-501, 1990.

MOLINA, R.; MASSICOTTE, H. B.; TRAPPE, J. M. Specificity phenomen in mycorrhizal simbioses: community-ecological consequences and pratical implications. In: ALLEN, M. F. (Ed.). Mycorrhizal functioning: an integrative plant-fungal process. London: Chapman \& Hall, 1992. p. 357-423.

OEHL, F.; SIEVERDING, E.; INEICHEN, K.; MÄDER, P.; WIEMKEN, A.; BOLLER, T. Distinct sporulation dynamics of arbuscular mycorrhizal fungal communities from different agroecosystems in long-term microcosms. Agriculture, Ecosystems \& Environment, Amsterdam, v. 134, n. 3-4, p. 257-268, 2009.

RONSHEIM, M. L. The effect of mycorrhizae on plant growth and reproduction varies with soil phosphorus and developmental stage. The American Midland Naturalist, Notre Dame, v. 167, n. 1, p. 28-39, 2012.

SAGGIN JUNIOR, O. J.; SILVA, E. M. R. Micorriza Arbuscular - papel, funcionamento e aplicação da simbiose. AQUINO, A. M.; ASSIS, R. L. (Ed.). Processos biológicos no sistema solo-planta. Brasília: Embrapa Informação Tecnológica, 2005. p. 101-104.

SIQUEIRA, J. O.; SAGGIN JÚNIOR, O. J. Dependency on arbuscular mycorrhizal fungi and responsiveness of some Brazilian native woody species. Mycorrhiza, New York, v. 11, n. 1, p. 245-255, 2001.

SMITH, S. E.; READ, D. J. Mycorrhizal symbiosis. $3^{\text {rd }}$ ed. London: Academic Press and Elsevier, 2008. 800 p.

SMITH, S. E.; SMITH, F. A.; JAKOBSEN, I. Mycorrhizal fungi can dominate phosphate supply to plants irrespective of growth responses. Plant Physiology, Washington, v. 133, n. 1, p. 16-20, 2003.

SUBRAMANIAN, K. S.; JEGAN, R. A.; GOMATHY, M.; VIJAYAKUMAR, S. Biochemical and nutritional responses of tripartite soybean-rhizobium-glomus association under low and high $\mathrm{P}$ fertilization. Madras Agricultural Journal, India, v. 98, n. 7-9, p. 224-228, 2011.

ZANGARO, W.; BONONI, V. L. R.; TRUFEN, S. B. Mycorrhizal dependency, inoculum potential and habitat preference of native woody species in South Brazil. Journal of Tropical Ecology, Cambridge, v. 16, n. 4, p. 603-624, 2000. 
